\title{
Evaluation of Immature Platelet Fraction in Lower Respiratory Tract Infections: A Retrospective Study
}

Vasiliki E. Georgakopoulou ${ }^{1,2}$, Dimitrios Mermigkis ${ }^{2}$, Konstantinos Mantzouranis ${ }^{2}$, Christos Damaskos ${ }^{3}$, ${ }^{4}$, Despoina Melemeni ${ }^{2}$, Eleni A. Alafaki ${ }^{5}$, Georgios Petsinis ${ }^{2}$, Nikolaos Garmpis ${ }^{6}$, Evgenia Karakou ${ }^{7}$, Anna Garmpi ${ }^{8}$, Agathi Lekkakou ${ }^{2}$, Pagona Sklapani ${ }^{9}$, Nikolaos Trakas ${ }^{7}$, Rea Chatzikyriakou ${ }^{5}$, Xanthi Tsiafaki $^{2}$

1. Pulmonology Department, Laiko General Hospital, Athens, GRC 2. 1st Pulmonology Department, Sismanogleio Hospital, Athens, GRC 3. Renal Transplantation Unit, Laiko General Hospital, Athens, GRC 4. N.S. Christeas Laboratory of Experimental Surgery and Surgical Research, Medical School, National and Kapodistrian University of Athens, Athens, GRC 5. Hematology, Sismanogleio Hospital, Athens, GRC 6. Second Department of Propedeutic Surgery, Laiko General Hospital, Medical School, National and Kapodistrian University of Athens, Athens, GRC 7. Biochemistry Department, Sismanogleio Hospital, Athens, GRC 8. First Department of Propedeutic Internal Medicine, Laiko General Hospital, Medical School, National and Kapodistrian University of Athens, Athens, GRC 9. Cytopathology Department, Mitera Hospital, Athens, GRC

Corresponding author: Vasiliki E. Georgakopoulou, vaso_georgakopoulou@hotmail.com

\section{Abstract}

\section{Introduction}

Immature platelet fraction (IPF) is a parameter of an automated hematologic analyzer and is related to platelet size and cytoplasmic RNA content. It reflects thrombopoiesis and is often used as the marker of platelet activity. IPF has been evaluated mostly in hematologic disorders and has also been evaluated in patients with gestational hypertension, sepsis, autoimmune diseases and in hospitalised patients with neutrophilia. Platelets, asides from the maintenance of hemostasis, release inflammatory mediators that can modify leukocyte and endothelial responses to various inflammatory stimuli. Lower respiratory tract infections are the leading cause of death from infections worldwide. The role of platelets in lower respiratory tract infections has been reported in many studies. IPF, which is related to platelet activation, has not been evaluated in patients with lower respiratory tract infections.

\section{Methods}

The study involved patients who fulfilled the criteria of community-acquired pneumonia (CAP) and aspiration pneumonia (AP). In addition, age and sex-matched healthy controls were involved. Whole blood samples were collected from healthy controls and from the patients on admission. The mean IPF\% and Creactive protein (CRP) levels were measured in patients with CAP, in patients with AP and in healthy controls. The mean IPF\% values in patients with infection were compared to mean IPF\% values in healthy

Received 07/01/2020 Review began $07 / 07 / 2020$ Review ended 07/08/2020 Published 07/16/2020

\section{() Copyright 2020}

Georgakopoulou et al. This is an open access article distributed under the terms of the Creative Commons Attribution License CC-BY 4.0., which permits unrestricted use, distribution, and reproduction in any medium, provided the original author and source are credited. controls. The mean IPF\% values were compared to mean CRP levels in patients with infection. Additionally, the mean IPF\% values in patients that died in the first 14 days were compared to the mean IPF\% values in patients that were alive. The statistical analysis of data was performed with the Statistical Package for the Social Sciences (SPSS) for Windows, Version 13.0 (SPSS Inc, Chicago, IL).

\section{Results}

The study population consisted of 45 patients ( 27 patients with CAP and 18 patients with AP), 27 males and 18 females, with a mean age of $72.11 \pm 16.4$ years and 39 healthy controls, 22 males and 17 females with a mean age of $64.2 \pm 14.8$ years. The mean CRP levels in patients with infection were $155.2 \pm 119.1 \mathrm{mg} / \mathrm{dl}$. The mean IPF\% value of patients with infection was $2.76 \pm 2.27$ and the mean IPF\% value of controls was $1.72 \pm$ $0.77(\mathrm{p}<0.006)$. The IPF\% value in patients with CAP was $2.55 \pm 2.02$ and in patients with AP $3.07 \pm 2.64$ ( $\mathrm{p}=$ 0.595). The mean IPF\% value in patients with infection had no linear correlation with CRP value in these patients $(r=0.076, p=0.62)$. The mean IPF\% value in all patients that died in the first 14 days was $3.75 \pm$ 2.44 and the mean IPF\% value in all patients alive was $2.35 \pm 2.11(\mathrm{p}=0.06)$. The mean IPF\% value in patients with CAP who died in the first 14 days of hospitalisation was $5.54 \pm 3.17$ and in patients with CAP who were alive was $1.87 \pm 0.72(\mathrm{p}=0.06)$. The mean IPF\% value in patients with AP who died was $2.63 \pm 0.85$ and in patients with AP who were alive was $3.41 \pm 3.51(\mathrm{p}=0.554)$.

\section{Conclusions}

Mean IPF\% value is greater in patients with lower respiratory tract infections, including CAP and AP, compared to healthy controls. There is no linear correlation between IPF values and CRP values in patients with lower respiratory tract infections. In addition, there is a difference in mean IPF\% value between patients who died in the first 14 days of hospitalisation compared to those who were alive, but not statistically significant. 
Categories: Infectious Disease, Pulmonology, Hematology

Keywords: platelets, respiratory infections, immature platelet fraction

\section{Introduction}

Immature platelet fraction (IPF) represents the percentage of platelets in circulation retaining RNA. It is an innovative parameter measuring reticulated platelets and is considered a rapid automated biomarker for differentiating cases of thrombocytopenia. It is calculated by flow cytometry or hematology analysers [1]. High IPF values are observed in conditions characterised by rapid platelet destruction and low IPF values are observed in disorders with low bone marrow activity [2].

IPF reference intervals are not clear. In a study in a large Danish population, the reference interval for IPF was $1.3 \%-9 \%$ and in another study in American population was reported that reference intervals for IPF were $0.5 \%-3.2 \%$ in men and $0.4 \%-3.0 \%$ in women $[3,4]$.

IPF has been evaluated in numerous diseases. IPF has been evaluated in hematologic disorders such as immune thrombocytopenic purpura in adults [5] and childhood immune thrombocytopenia [6]. IPF levels have been found significantly higher in patients with gestational hypertension compared to controls [7]. IPF has been reported to have a comparable efficiency to C-reactive protein (CRP) in discriminating patients who had sepsis and those who had not and to be predicting the risk of developing sepsis in patients admitted to intensive care unit [8]. IPF could be used as a screening tool for bacterial infection in hospitalised patients with neutrophilia with significant correlation to blood culture positivity [9]. In addition, the role of IPF as a biomarker in patients with autoimmune diseases has been reviewed [1].

Platelets have a major role in the maintenance of hemostasis. However, they release several inflammatory mediators, not related to hemostasis. These mediators modify leukocyte and endothelial responses to various inflammatory stimuli. Platelets interact with neutrophlis, lymphocytes, monocytes and endothelial cells and therefore have a significant role in inflammation and immune responses [10].

Platelets contain several organelles such as mitochondria, lysosomes and peroxisomes, and numerous intracellular immune mediators stored in granules and vesicles. In addition, platelets express considerable membrane receptors and contain cytoplasmic mRNA, which can synthesize a few proteins. These receptors and proteins allow platelets to interact with leukocytes and endothelial cells. Thus, platelets can regulate immune responses at the sites of platelet activation systemically. Platelet receptors and the molecules stored in platelet granules regulate platelets functions. There are three main types of platelet granules: $\alpha$ granules, dense granules and lysosomal granules. $\alpha$-granules are the most abundant and largest granules and they contain close to 300 proteins, including chemokines, such as P-selectin, CXCL1, CXCL5, CXCL7, CXCL12, macrophage inflammatory protein (MIP)-1 $\alpha$, regulated on activation normal T expressed and secreted (RANTES) and platelet factor 4 (PF4). Dense granules are smaller, less abundant and store small molecules, such as ADP, ATP, inorganic polyphosphate, histamine and serotonin, while lysosomal granules are scattered and contain proteases and glycosidases [11].

When platelets are stimulated, granules undergo exocytosis and release their molecules with immunomodulating properties into the extracellular space. In addition, molecules found on the inner granule membrane become surface-expressed [11]. Activated platelets also release interleukin-1 $\beta$ (IL-1 $\beta$ ), which is not granule-stored but produced upon platelet activation [12].

Lower respiratory tract infections are the leading cause of death from infections worldwide [13]. The role of platelets in lower respiratory tract infections has been reported in many studies. The activation of platelets by the S.pneumoniae, the most common cause of community-acquired pneumonia (CAP), in vitro, has been reported [14]. The mechanisms of platelet activation by the pneumococcus and the role of platelets in CAP have been described [15]. It has been reported that the presence of phage protein pblB on the pneumococcal chromosome probably results to increased mortality in patients with an invasive S. pneumoniae infection, which may be explained by enhanced platelet activation [16].

Mean platelet volume (MPV), which is another parameter related to platelet activation, has been evaluated in lower respiratory tract infections [17]. Elevated MPV strongly predicts in-hospital and long-term mortality in patients hospitalised for CAP, while changes in MPV during intensive care unit admission can probably be used as a prognostic index of mortality in intensive care unit patients with pneumonia [18,19].

However, IPF, which also is related to platelet activation, has not been evaluated in patients with lower respiratory tract infections. We aim to evaluate IPF in patients with lower respiratory tract infections.

\section{Materials And Methods}

The study involved patients who fulfilled the criteria of CAP and aspiration pneumonia (AP). The exclusion criteria were: patients $<18$ years old, patients with a history of solid tumors or hematological malignancy, 


\section{Cureus}

patients suffering from disease or receiving therapy that suppresses the bone marrow activity, patients receiving antiplatelet therapy and patients with platelet counts of less than $150 \mathrm{x} 10^{3} / \mu \mathrm{l}$. In addition, age and sex-matched healthy controls without infection, and with platelet counts of more than $150 \times 10^{3} / \mu l$, were involved. Whole blood samples were collected from healthy controls and from the patients on admission. The mean IPF\% and CRP levels were measured in patients with CAP, in patients with AP and in healthy controls. IPF was measured in automated Sysmex XE 2100 hematology analyzer. Serum levels of CRP were determined by immunoturbidimetric assay on Roche Cobas 6000 c501 analyzer. Reference range for IPF\% in our laboratory is $1 \%-5 \%$ and normal values for CRP are values less than $6 \mathrm{mg} / \mathrm{dl}$. The mean IPF\% values in patients with lower respiratory infection were compared to mean IPF\% values in healthy controls. The IPF\% values were compared to CRP levels in patients with infection. Additionally, the mean IPF\% values in patients who died in the first 14 days of hospitalisation were compared to the IPF\% values in patients that were alive. The statistical analysis of data was performed with the Statistical Package for the Social Sciences (SPSS) for Windows, Version 13.0 (SPSS Inc., Chicago, IL). Continuous variables were tested for normality of distribution by the Kolmogorov-Smirnov test. For normally distributed values, descriptive results are presented as mean (standard deviation). The statistical control for normal values performed with: (a) independent samples $\mathrm{T}$ test for normally distributed data, (b) one-way ANOVA and (c) Pearson $\mathrm{x}^{2}$ for control of independence of variables. All p-values were two-sided and 5\% was chosen as the level of statistical significance.

\section{Results}

The study population consisted of 45 patients ( 27 patients with CAP and 18 patients with AP), 27 males and 18 females, with a mean age of $72.11 \pm 16.4$ years and 39 healthy controls, 22 males and 17 females with a mean age of $64.2 \pm 14.8$ years (Table 1 ).

\begin{tabular}{|c|c|c|c|c|c|c|}
\hline & C, $n=39$ & $A P, n=18$ & CAP, $n=27$ & C versus AP & C versus CAP & AP versus CAP \\
\hline Gender (males/females) & $22 / 17$ & $11 / 7$ & 16/11 & 0.738 & 0.818 & 0.901 \\
\hline Age(years) (SD) & $64.2(14.8)$ & 77.5 (13.3) & $69.7(17.1)$ & $<0.01$ & 0.339 & 0.238 \\
\hline CRP (mg/dl) (SD) & $3.28(1.05)$ & 188.3 (115.9) & $133.2(118.1)$ & $<0.0001$ & $<0.0001$ & 0.092 \\
\hline IPF (\%) (SD) & $1.72(0.77)$ & 3.07 (2.64) & $2.55(2.02)$ & $<0.023$ & 0.148 & 0.595 \\
\hline Status (alive/dead) & $39 / 0$ & $10 / 8$ & $22 / 5$ & $<0.0001$ & $<0.0001$ & 0.060 \\
\hline
\end{tabular}

\section{TABLE 1: Characteristics of the study population}

C, controls; AP, aspiration pneumonia; CAP, community-acquired pneumonia; IPF, immature platelet fraction. Status: alive/dead in the first 14 days of hospitalisation

The mean CRP levels in patients with lower respiratory tract infections were $155.2 \pm 119.1 \mathrm{mg} / \mathrm{dl}$ (Table 2). The mean IPF\% value of patients with lower respiratory tract infections was $2.76 \pm 2.27$ and the mean IPF\% value of controls was $1.72 \pm 0.77(\mathrm{p}<0.006)$ (Table 2; Figure 1$)$.

\begin{tabular}{|c|c|c|c|}
\hline & $C, n=39$ & Patients, $n=45$ & C versus patients \\
\hline IPF (\%) (SD) & $1.72(0.77)$ & $2.76(2.27)$ & $<0.006$ \\
\hline CRP (mg/dl) (SD) & 3.28(1.05) & 155.2(119.1) & $<0.0001$ \\
\hline
\end{tabular}

TABLE 2: Mean IPF\% and CRP values in patients with infection and healthy controls

C, controls; IPF, immature platelet fraction; CRP, C-reactive protein 


\section{Cureus}

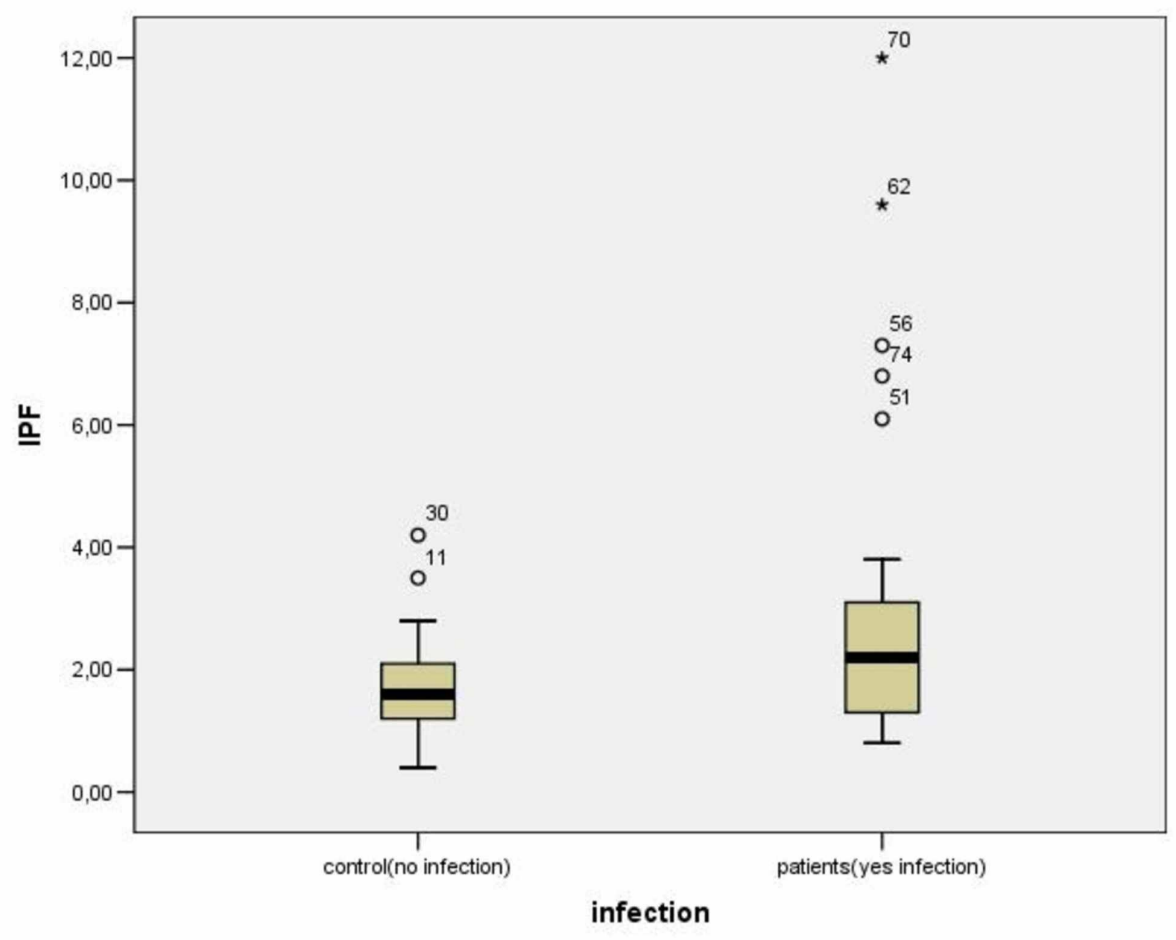

FIGURE 1: Comparison of IPF\% values between infected and healthy controls

11, 30, 51, 56, 74: outliers; 62,70 : extreme values. IPF, immature platelet fraction

The mean IPF\% value in patients with CAP was $2.55 \pm 2.02$ and in patients with AP $3.07 \pm 2.64(\mathrm{p}=0.595)$ (Figure 2). The IPF\% values in patients with lower respiratory tract infections had no linear correlation with CRP values in these patients $(r=0.076, p=0.62)$. (Table 3; Figure 3). Thirteen patients died in the first 14 days of hospitalisation [eight patients (61.5\%) with AP and five patients (18.5\%) with CAP (Figure 4 ). The mean IPF\% value in all patients that died in the first 14 days was $3.75 \pm 2.44$ and the mean IPF\% value in all patients that were alive was $2.35 \pm 2.11(\mathrm{p}=0.06$ ) (Table 4 ; Figure 5). The mean IPF\% value in patients with CAP who died in the first 14 days of hospitalisation was $5.54 \pm 3.17$ and in patients with CAP who were alive was $1.87 \pm 0.72(p=0.06)$. The mean IPF\% value in patients with AP who died in the first 14 days of hospitalisation was $2.63 \pm 0.85$ and in patients with AP who were alive was $3.41 \pm 3.51(\mathrm{p}=0.554)$ (Table 5). 


\section{Cureus}

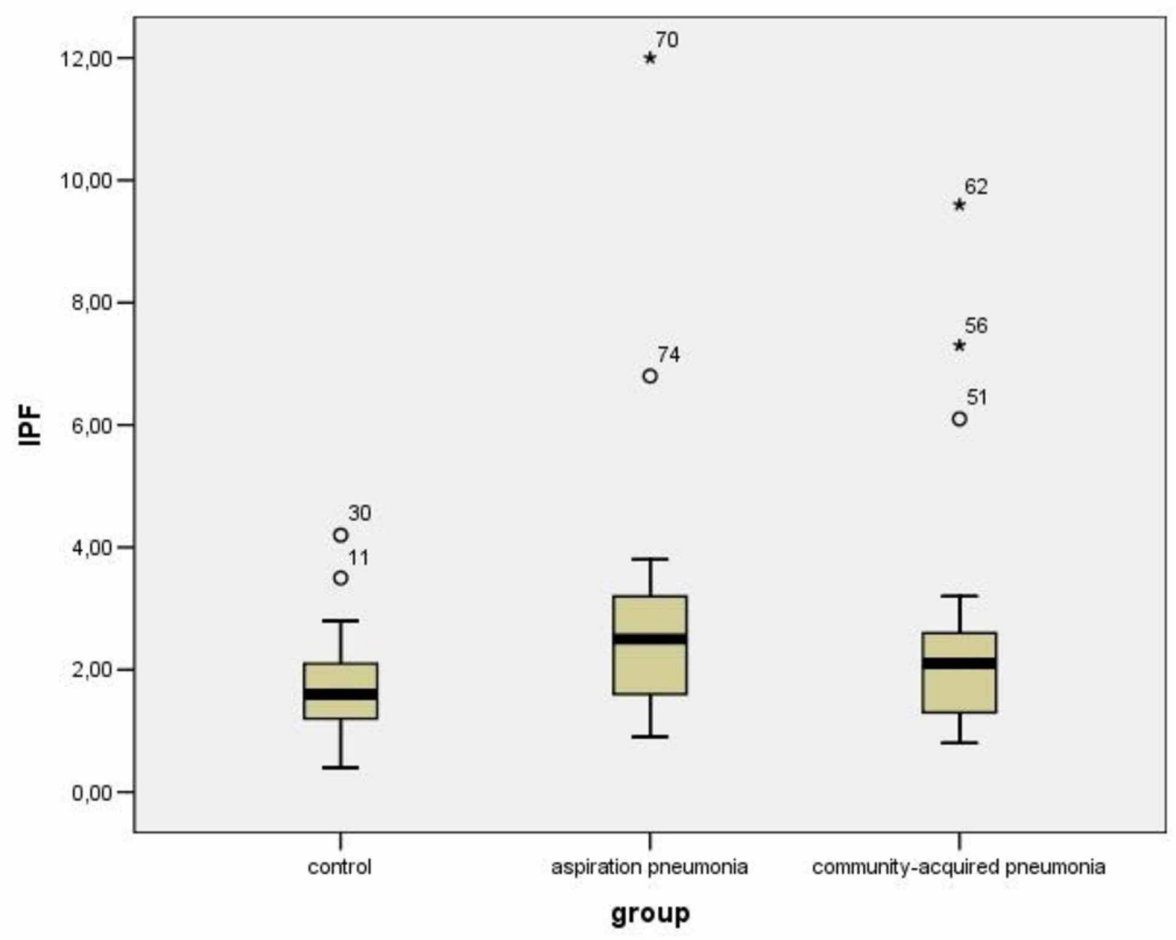

FIGURE 2: Comparison of IPF\% values between groups

$11,30,51,56,74$ : outliers; 62,70 : extreme values. IPF, immature platelet fraction

\begin{tabular}{|l|l|l|l|}
\hline REGRESSION & $r$ & $r^{2}$ & $p$ \\
\hline CRP $(\mathrm{mg} / \mathrm{dl})=2.981-0.001(\operatorname{IPF}(\%))$ & 0.076 & 0.006 & 0.620 \\
\hline
\end{tabular}

TABLE 3: Correlation between IPF\% values and CRP values in patients with lower respiratory tract infections

CRP, C-reactive protein; IPF, immature platelet fraction 


\section{Cureus}

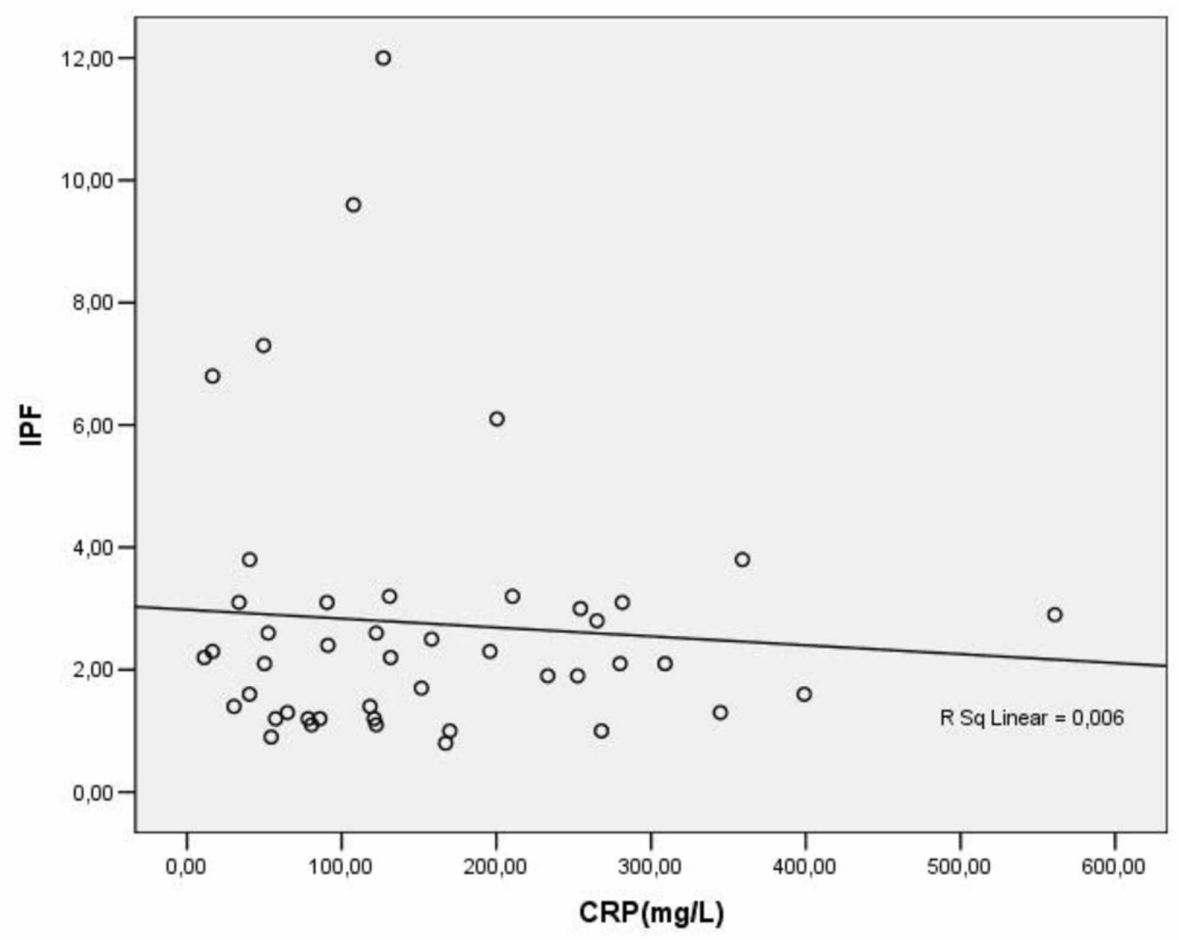

FIGURE 3: Correlation between IPF\% and CRP values in infected patients.

CRP, C-reactive protein; IPF, immature platelet fraction

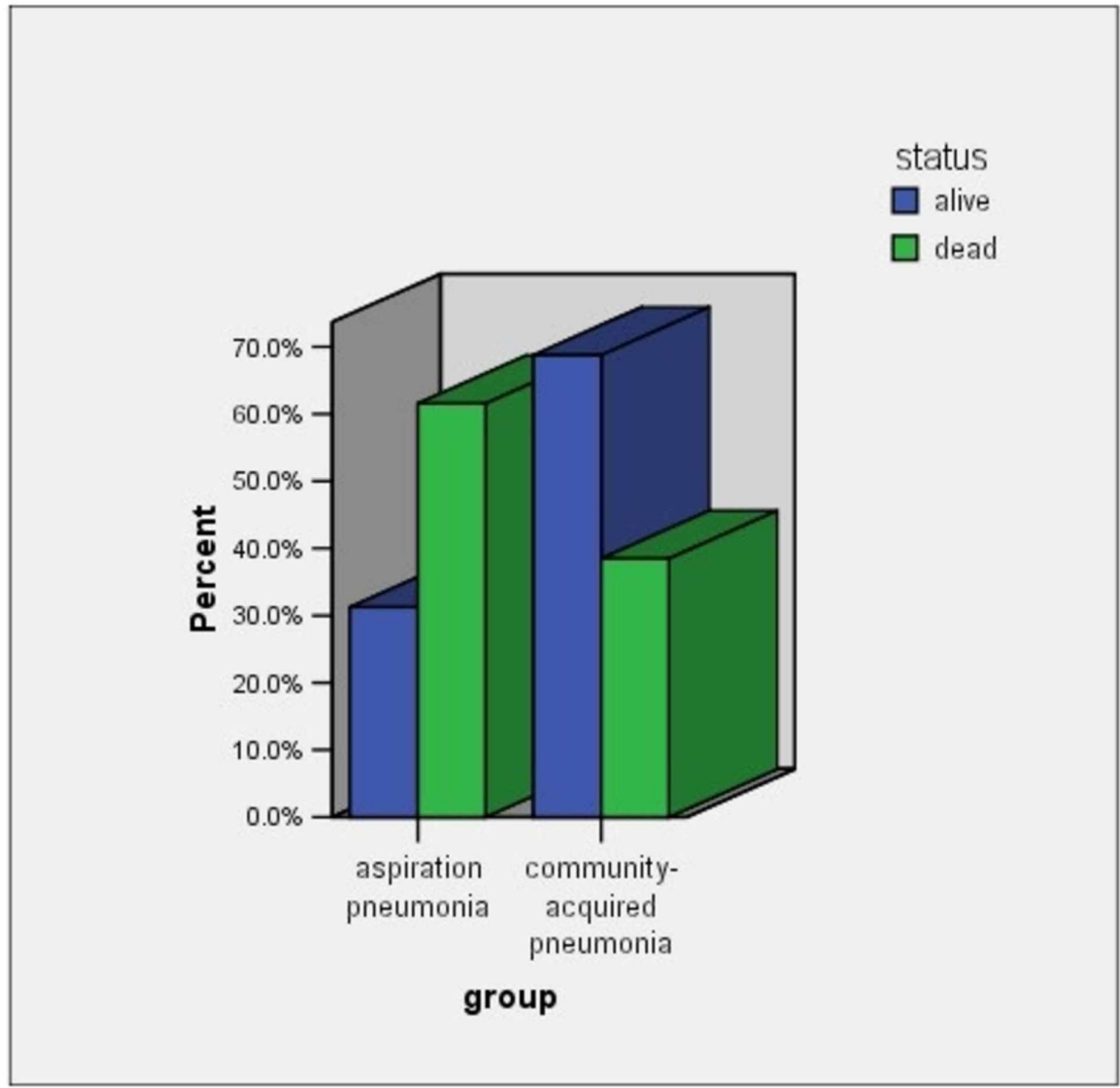

FIGURE 4: People alive/dead in each group of infection in the first 14 


\section{Cureus}

\section{days of hospitalisation}

\begin{tabular}{|c|c|c|c|}
\hline Status & Alive, $\mathrm{n}=32$ & Dead, $n=13$ & Alive versus Dead \\
\hline IPF (\%) (SD) & $2.35(2.11)$ & $3.75(2.44)$ & 0.06 \\
\hline CRP (mg/dl) (SD) & 141.8 (124.2) & 188.3 (102.3) & 0.239 \\
\hline
\end{tabular}

TABLE 4: Mean IPF\% values in dead and alive patients in the first 14 days of hospitalisation CRP, C-reactive protein; IPF, immature platelet fraction

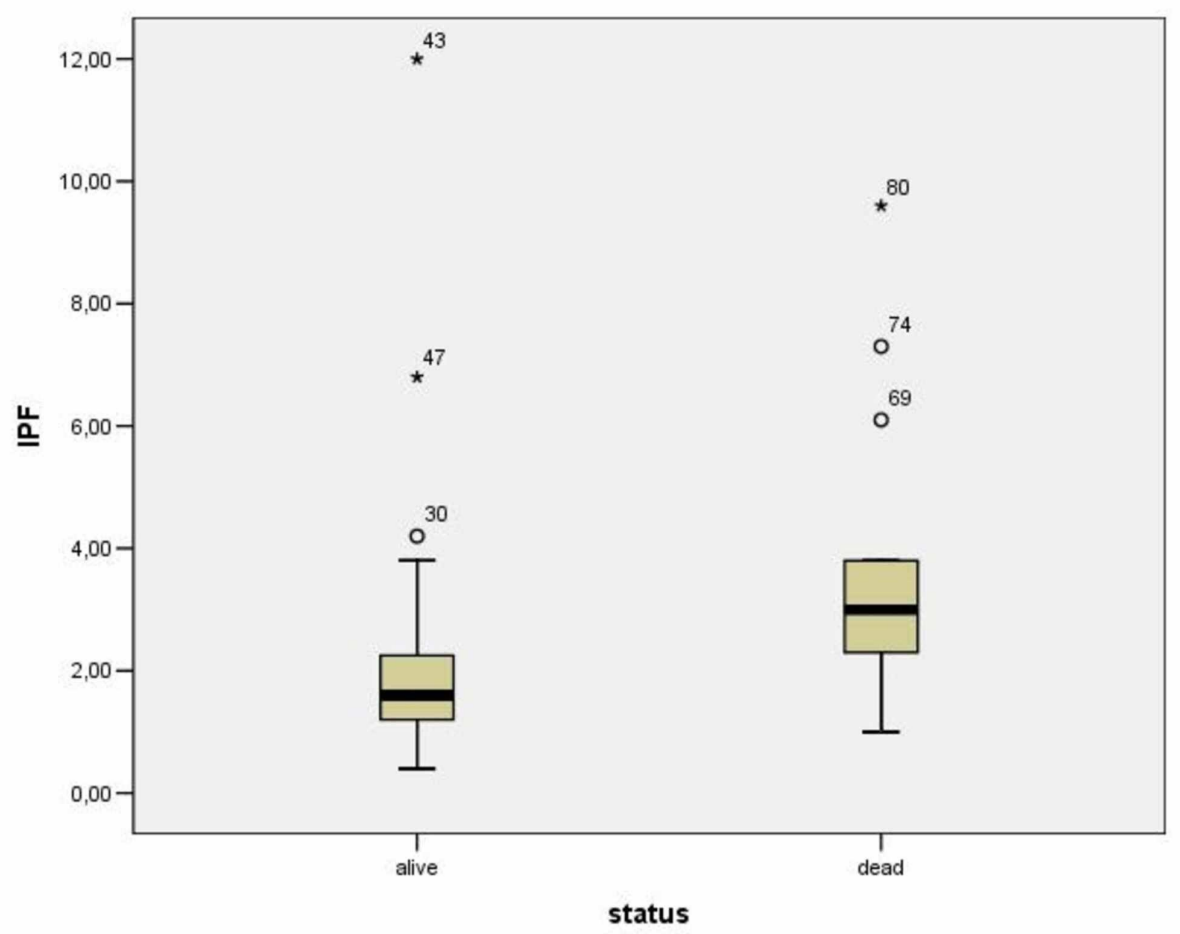

FIGURE 5: Comparison of IPF\% values between dead and alive patients in the first 14 days

30, 69,74 : outliers; $43,47,80$ : extreme values; IRF, immature platelet fraction 


\section{Cureus}

\begin{tabular}{|c|c|c|c|c|}
\hline & Status & Alive, $n=10$ & Dead, $\mathbf{n}=8$ & Alive versus Dead \\
\hline \multirow[t]{3}{*}{ AP } & IPF (\%) (SD) & $3.41(3.51)$ & 2.63(0.85) & 0.554 \\
\hline & CRP (mg/dl) (SD) & $158.6(119.9)$ & $225.5(106.3)$ & 0.235 \\
\hline & Status & Alive $\mathrm{n}=22$ & Dead $n=5$ & Alive versus Dead \\
\hline \multirow[t]{2}{*}{ CAP } & IPF (\%) (SD) & $1.87(0.72)$ & $5.54(3.17)$ & 0.06 \\
\hline & CRP (mg/dl) (SD) & 134.1(128.2) & 128.9(66.7) & 0.931 \\
\hline \multicolumn{5}{|c|}{$\begin{array}{l}\text { TABLE 5: Mean IPF\% values and mean CRP values in patients with AP and CAP, dead and alive in } \\
\text { the first } 14 \text { days of hospitalisation }\end{array}$} \\
\hline \multicolumn{5}{|c|}{ AP, aspiration pneumonia; CAP, community-acquired pneumonia; CRP, C-reactive protein; IPF, immature platelet fraction } \\
\hline
\end{tabular}

\section{Discussion}

According to our results, there was a difference in mean IPF\% value between patients with AP compared to the patients with CAP but no statistically significant. However, there was a statistically significant difference in mean IPF\% value between all patients with respiratory infections and healthy controls, indicating increased bone marrow platelet production in infected patients. Platelets are anucleate blood cells, with intimate relationship with the lungs, which transit the pulmonary vessels and are present in alveolar capillaries. Megakaryocytes, the cells that are the precursors of platelets, are present in the mammalian lungs. There is evidence that the lungs are sites of thrombopoiesis, and that human lungs are reservoirs for platelets and release them in response to specific stimuli [20]. In addition, there is evidence that platelets regulate vascular permeability in the lungs and the barrier function of the alveolar capillaries. Recent studies indicate that platelets have a key role in pulmonary immune responses and integrity but that these cells can also contribute to injury in lung diseases [20].

As mentioned in the introduction, the platelet activation related to S. pneumoniae has been studied [14]. In addition, platelet activation associated with Staphylococcus aureus and Klebsiella pneumoniae-induced sepsis has been studied in numerous animal models of pulmonary bacterial infections [21]. There is evidence that platelets have an important role in the immune response to respiratory bacterial infection with $\mathrm{P}$. aeruginosa. It has been reported that experimentally-induced platelet depletion leads to increased pulmonary bacterial growth and systemic bacterial dissemination indicating that platelets play a significant role in preventing bacterial lung infections [22]. Besides bacterial infections, the role of platelets in lower respiratory tract viral infections has been reported. Schrottmaier et al. described the interaction between platelets and leukocytes and the subsequent alteration in cytokine production as a possible mechanism involved in defense against the respiratory syncytial virus (RSV) [23].

CRP was identified in 1930 and is considered to be an acute-phase protein, indicating infectious or inflammatory disorders [24]. CRP has been studied as a diagnostic tool and as a biomarker for disease severity in lower tract infections and as a marker for prognosis in patients with CAP [25-27]. However, in our study, the IPF\% values in patients with lower respiratory tract infections had no linear correlation with CRP values in these patients $(r=0.076, p=0.62)$.

Liu et al. studied the correlation between the percentage of immature platelets and infection in 190 patients with a body temperature $>37.3^{\circ} \mathrm{C}$ or $<36^{\circ} \mathrm{C}$ and suspicious of infection, who were hospitalized and concluded that the sensitivity and specificity for diagnosing infection were, respectively, $91.78 \%$ and $93.18 \%$ when IPF\% and CRP were used in combination [28]. IPF\% changed dynamically during the course of the infection and recovered to lower than $5.5 \%$ at two to seven days before the body temperature was normal.

In our study, there was a difference in mean IPF\% value between patients that were alive and patients who died in the first 14 days of hospitalisation, with greater mean IPF \% value noticed among patients that died in the first 14 days of hospitalisation, but not statistically significant. These results indicate a greater platelet bone marrow production in patients who died. The association between platelet counts and mortality in patients with lower tract infections has been reported. Mirsaeidi et al. in their retrospective cohort study concluded that platelet abnormalities are associated with mortality in patients hospitalized with CAP and evaluating an initial complete blood count test in patients with CAP, an abnormal platelet count is a better predictor of outcome than an abnormal leukocyte count [29]. Brogly et al. in their multicentre observational study reported that thrombocytopenia is an independent predictor of mortality in patients with severe CAP [30].

The study has some limitations. The fact that all the patients included in this study were enrolled in a single 
medical center and the relatively small sample size of patients lead to limitations in the generalizability of the results. A further well-controlled, prospective study is needed to clarify the relationship between IPF and lower respiratory tract infections.

\section{Conclusions}

Mean IPF\% values are greater in patients with lower respiratory tract infections, including CAP and AP, compared to healthy controls. There is no linear correlation between IPF values and CRP values in patients with lower respiratory tract infections. In addition, there is no statistically significant difference in mean IPF\% value between patients that died in the first 14 days of hospitalisation compared to those who were alive. Based on our study, we cannot conclude that IPF is a reliable inflammatory marker or prognostic factor for lower respiratory tract infections. To our knowledge, the current study is the first to evaluate IPF in patients with lower respiratory tract infections. Further studies are needed to establish the role of IPF as a biomarker in these patients.

\section{Additional Information \\ Disclosures}

Human subjects: Consent was obtained by all participants in this study. Animal subjects: All authors have confirmed that this study did not involve animal subjects or tissue. Conflicts of interest: In compliance with the ICMJE uniform disclosure form, all authors declare the following: Payment/services info: All authors have declared that no financial support was received from any organization for the submitted work. Financial relationships: All authors have declared that they have no financial relationships at present or within the previous three years with any organizations that might have an interest in the submitted work. Other relationships: All authors have declared that there are no other relationships or activities that could appear to have influenced the submitted work.

\section{References}

1. Schmoeller D, Picarelli MM, Munhoz TP, Poli de Figueiredo CE, Staub H: Mean platelet volume and immature platelet fraction in autoimmune disorders. Front Med. 2017, 4:146. 10.3389/fmed.2017.00146

2. Jung H, Jeon HK, Kim HJ, Kim SH: Immature platelet fraction: establishment of a reference interval and diagnostic measure for thrombocytopenia. Korean J Lab Med. 2010, 30:451-459. 10.3343/kjlm.2010.30.5.451

3. Joergensen MK, Bathum L: Reference intervals for mean platelet volume and immature platelet fraction determined on a sysmex XE5000 hematology analyzer. Scand J Clin Lab Invest. 2016, 76:172-176. $10.3109 / 00365513.2015 .1124448$

4. Ibrahim H, Nadipalli S, Usmani S, DeLao T, Green L, Kleiman NS: Detection and quantification of circulating immature platelets: agreement between flow cytometric and automated detection. J Thromb Thrombolysis . 2016, 42:77-83. 10.1007/s11239-016-1338-3

5. Naz A, Mukry SN, Shaikh MR, Bukhari AR, Shamsi TS: Importance of immature platelet fraction as predictor of immune thrombocytopenic purpura. Pak J Med Sci. 2016, 32:575-579. 10.12669/pjms.323.9456

6. Moneam Adly AA, Ragab IA, Rahman Ismail EA, Farah MM: Evaluation of the immature platelet fraction in the diagnosis and prognosis of childhood immune thrombocytopenia. Platelets. 2015, 26:645-650. 10.3109/09537104.2014.969220

7. Moraes D, Munhoz TP, Pinheiro da Costa BE, et al.: Immature platelet fraction in hypertensive pregnancy . Platelets. 2016, 27:333-337. 10.3109/09537104.2015.1101060

8. Buoro S, Manenti B, Seghezzi M, et al.: Innovative haematological parameters for early diagnosis of sepsis in adult patients admitted in intensive care unit. J Clin Pathol. 2018, 71:330-335. 10.1136/jclinpath-2017204643

9. Di Mario A, Garzia M, Leone F, Arcangeli A, Pagano L, Zini G: Immature platelet fraction (IPF) in hospitalized patients with neutrophilia and suspected bacterial infection. J Inf. 2009, 59:201-206. 10.1016/j.jinf.2009.07.007

10. Thomas MR, Storey RF: The role of platelets in inflammation . Thromb Haemostasis. 2015, 114:449-458. 10.1160/TH14-12-1067

11. Gomez-Casado C, Villaseñor A, Rodriguez-Nogales A, Bueno JL, Barber D, Escribese MM: Understanding platelets in infectious and allergic lung diseases. Int J Mol Sci. 2019, 20:1730. 10.3390/ijms20071730

12. Lindemann S, Tolley ND, Dixon DA, McIntyre TM, Prescott SM, Zimmerman GA, Weyrich AS: Activated platelets mediate inflammatory signaling by regulated interleukin 1beta synthesis. J Cell Biol. 2001, 154:485490. 10.1083 /jcb. 200105058

13. GBD 2015 LRI Collaborators: Estimates of the global, regional, and national morbidity, mortality, and aetiologies of lower respiratory tract infections in 195 countries: a systematic analysis for the Global Burden of Disease Study 2015. Lancet Inf Dis. 2017, 17:1133-1161. 10.1016/S1473-3099(17)30396-1

14. Guckian JC: Effect of pneumococci on blood clotting, platelets, and polymorphonuclear leukocytes . Infect Immun. 1975, 12:910-918.

15. Anderson R, Feldman C: Review manuscript: mechanisms of platelet activation by the pneumococcus and the role of platelets in community-acquired pneumonia. J Inf. 2017, 75:473-485. 10.1016/j.jinf.2017.09.013

16. Tunjungputri RN, Mobegi FM, Cremers AJ, et al.: Phage-derived protein induces increased platelet activation and is associated with mortality in patients with invasive pneumococcal disease. mBio. 2017, 8:01984-16. 10.1128/mBio.01984-16

17. Park Y, Schoene N, Harris W: Mean platelet volume as an indicator of platelet activation: methodological issues. Platelets. 2002, 13:301-306. 10.1080/095371002220148332

18. Gorelik O, Tzur I, Barchel D, et al.: A rise in mean platelet volume during hospitalization for community- 
acquired pneumonia predicts poor prognosis: a retrospective observational cohort study. BMC Pulm Med. 2017, 17:137. 10.1186/s12890-017-0483-6

19. Lee JH, Park MA, Han SW, Hwang JJ, Park SH, Park SY: An increase in mean platelet volume during admission can predict the prognoses of patients with pneumonia in the intensive care unit: a retrospective study. Plos One. 2018, 13:0208715. 10.1371/journal.pone.0208715

20. Weyrich AS, Zimmerman GA: Platelets in lung biology. Annu Rev Physiol. 2013, 75:569-591. 10.1146/annurev-physiol-030212-183752

21. Youssefian T, Drouin A, Masse JM, Guichard J, Cramer EM: Host defense role of platelets: engulfment of HIV and Staphylococcus aureus occurs in a specific subcellular compartment and is enhanced by platelet activation. Blood. 2002, 99:4021-4029. 10.1182/blood-2001-12-0191

22. Amison RT, O'Shaughnessy BG, Arnold S, et al.: Platelet depletion impairs host defense to pulmonary infection with pseudomonas aeruginosa in mice. Am J Respir Cell Mol Biol. 2018, 58:331-340. 10.1165/rcmb.2017-00830C

23. Schrottmaier WC, Kral JB, Badrnya S, Assinger A: Aspirin and P2Y12 Inhibitors in platelet-mediated activation of neutrophils and monocytes. Thromb Haemost. 2015, 114:478-489. 10.1160/TH14-11-0943

24. Clyne B, Olshaker JS: The C-reactive protein. J Emerg Med. 1999, 17:1019-1025. 10.1016/s07364679(99)00135-3

25. Chalmers JD, Singanayagam A, Hill AT : C-reactive protein is an independent predictor of severity in community-acquired pneumonia . Am J Med . 2008, 121:219-225. 10.1016/j.amjmed.2007.10.033

26. Andersen SB, Baunbæk Egelund G, Jensen AV, Petersen PT, Rohde G, Ravn P: Failure of CRP decline within three days of hospitalization is associated with poor prognosis of Community-acquired Pneumonia. Infect Dis. 2017, 49:251-260. 10.1080/23744235.2016.1253860

27. Guo S, Mao X, Liang M: The moderate predictive value of serial serum CRP and PCT levels for the prognosis of hospitalized community-acquired pneumonia. Respir Res. 2018, 19:193. 10.1186/s12931-018-0877-X

28. Liu QH, Song MY, Yang BX, Xia RX: Clinical significance of measuring reticulated platelets in infectious diseases. Medicine. 2017, 96:e9424. 10.1097/MD.0000000000009424

29. Mirsaeidi M, Peyrani P, Aliberti S, et al.: Thrombocytopenia and thrombocytosis at time of hospitalization predict mortality in patients with community-acquired pneumonia. Chest. 2010, 137:416-420. 10.1378/chest.09-0998

30. Brogly N, Devos P, Boussekey N, Georges H, Chiche A, Leroy O: Impact of thrombocytopenia on outcome of patients admitted to ICU for severe community-acquired pneumonia. J Infect. 2007, 55:136-140. 10.1016/j.jinf.2007.01.011 